\title{
A Comprehensive Modeling of a Three-Phase Voltage Source PWM Converter
}

\author{
Juan Segundo-Ramírez, ${ }^{1}$ Rafael Peña-Gallardo, ${ }^{1}$ Aurelio Medina, ${ }^{2}$ \\ Ciro Núñez-Gutiérrez, ${ }^{1}$ and Nancy Visairo-Cruz ${ }^{1}$ \\ ${ }^{1}$ Facultad de Ingeniería, Universidad Autónoma de San Luis Potosí, Manuel Nava No. 8, Zona Universitaria, \\ 78290 San Luis Potosí, SLP, Mexico \\ ${ }^{2}$ Facultad de Ingeniería Eléctrica, División de Estudios de Posgrado, Universidad Michoacana de San Nicolás de Hidalgo, \\ Ciudad Universitaria, 58030 Morelia, MICH, Mexico \\ Correspondence should be addressed to Rafael Peña-Gallardo; rafael.pena@uaslp.mx
}

Received 22 December 2014; Revised 29 April 2015; Accepted 3 May 2015

Academic Editor: Ivanka Stamova

Copyright ( 2015 Juan Segundo-Ramírez et al. This is an open access article distributed under the Creative Commons Attribution License, which permits unrestricted use, distribution, and reproduction in any medium, provided the original work is properly cited.

This contribution reports the development of a time domain model of a three-phase voltage source converter (VSC) that can be used in the transient and steady state analysis of nonlinear power systems including their associated closed-loop control schemes. With this proposed model, the original discontinuous nonlinear power system can be transformed into a continuous system, while keeping the underlying harmonic nature of the VSC and avoiding typical and undesirable numerical problems associated with the large derivatives during the switching transitions. The development of this model was based on the dynamic Fourier series of the switching functions under a sinusoidal PWM modulation scheme, which require the calculation of the switching instants at each integration step; the switching instants and the dynamic Fourier series coefficients are calculated by explicit mathematical formulas. The proposed model of the VSC is suitable for the fast computation of the periodic steady state solution through the application of Newton method. Simulations were carried out in order to illustrate the benefits of the proposed VSC model.

\section{Introduction}

This contribution describes a time domain model of the voltage source converter (VSC) that can be used in the transient and steady state analysis of nonlinear power systems including linear or nonlinear control schemes. With the proposed model, the original discontinuous nonlinear power system can be transformed into a continuous system while keeping the underlying discontinuous nature of the VSC and avoiding undesirable numerical problems associated with the large derivatives during the switching transitions. Additionally, the computation of the periodic steady state solution is obtained with a Newton method; in this paper, an enhanced numerical differentiation method is used [1].
The VSC is the heart of many components in industrial applications due to its modular design, controllability, and the ability to build some multilevel topologies for low and high power applications $[2,3]$, high voltage direct current (HVDC) transmission system [4], electric motor speed drives, interconnection of wind energy systems, and flexible AC transmission systems (FACTS) [5], among others. For these reasons, the three-phase VSC is the common building block in FACTS technologies, custom power equipment, active filters, variable speed drives, PWM rectifiers, HVDC links, and power electronic interfaces for interconnection of renewable energy sources. However, the analyses of electric networks including VSC-based components still represent important challenges, since the VSC-based components are in general nonlinear and incorporate both continuous and discontinuous time dynamics and discrete time events. 


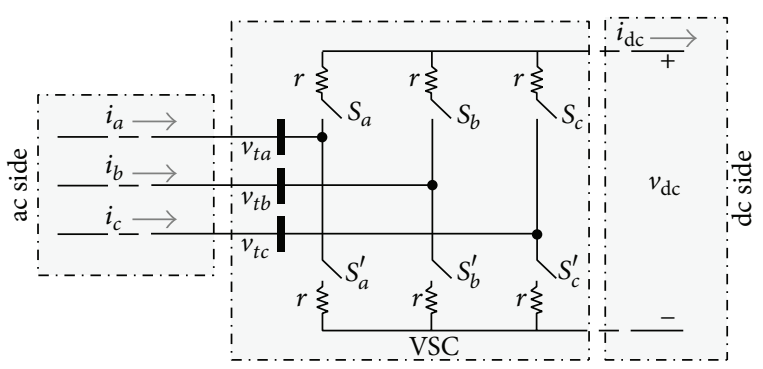

FIGURE 1: VSC circuit with ideal switches.

Several models have been presented in order to describe more accurately the VSC: in [6] two mathematical representations of the three-phase VSC for transient and steady state solutions of nonlinear electric systems are proposed. The three-phase VSC models in $a b c$-frame proposed in [7] can be used in a Newton-Raphson formulation for the fast computation of the periodic steady state solution and stability assessment of power systems with nonlinear loads and control systems, as demonstrated in [8-12]. In a previous contribution, an exact model for VSC in $\alpha \beta$ frame is presented in [13]. Reference [14] presents a direct harmonic method in the $\alpha \beta$-frame for the computation of the characteristic and uncharacteristic harmonic components of the VSC. In [15], an iterative method based on a hybrid time/frequency-domain approach is proposed to compute the steady state of a PWM VSC in the $\alpha \beta$-frame with a closed-loop controller. In these previous contributions [1315], the models are only limited to linear switched networks and also present some drawbacks for VSC with closed-loop control; that is, the quadratic convergence characteristics of the Newton-Raphson method for the fast computation of the periodic steady state solution are degraded. Reference [16] deals with a time domain method using a modified Newton method for only one VSC with $d q$ control; however, the size of the resulting system increases with the switching frequency and this approach does not address the stability problem.

This contribution deals with a powerful model of the VSC based on the Fourier series for time domain simulation of nonlinear power systems including VSC-based components considering large time steps. This model will be described in detail in the following sections.

\section{Algebraic Model of the VSC}

For reference, Figure 1 shows the circuit representation of the well-known three-phase two-level VSC. This VSC contains six bidirectional switches; each bidirectional switch is an arrangement of an IGBT (GTO or MOSFET) in antiparallel with a diode. In power system analysis, the engineers use the ideal switch model to represent semiconductors [17]. Using the ideal switch model, the original continuous nonlinear dynamic system that represents the VSC becomes a set of linear algebraic equations. The bidirectional switching function is identified by $S_{x}$ and $S_{x}^{\prime}$ for each phase $(x=$ $a, b, c)$, which can be on or off, $r$ is the switch-on state resistance, and $S_{x}$ is 1 or 0 , corresponding to the on and off states of the switch, respectively. In addition, $S_{x}$ and $S_{x}^{\prime}$ are complementary; that is, $S_{x}+S_{x}^{\prime}=1$.

The voltage to ground at the ac side of the VSC is given by

$$
\begin{aligned}
& v_{t a}=r i_{a}+v_{\mathrm{dc}} S_{a}-\frac{v_{\mathrm{dc}}\left(S_{a}+S_{b}+S_{c}\right)}{3}, \\
& v_{t b}=r i_{b}+v_{\mathrm{dc}} S_{b}-\frac{v_{\mathrm{dc}}\left(S_{a}+S_{b}+S_{c}\right)}{3}, \\
& v_{t c}=r i_{c}+v_{\mathrm{dc}} S_{c}-\frac{v_{\mathrm{dc}}\left(S_{a}+S_{b}+S_{c}\right)}{3} .
\end{aligned}
$$

The switching functions $S_{a}, S_{b}$, and $S_{c}$ are square waveforms and take values of 0 for some intervals of time every fundamental period and values of 1 for the rest of the fundamental period. The time instants when the switching functions change from 1 to 0 and vice versa are named switching times. Please notice in (1) that the relationship between the $\mathrm{dc}$ side and the ac side of the VSC is purely algebraic; thus, the voltage variations in one side are instantaneously seen for the other side and vice versa. The mathematical representation (1) allows any modulation technique. In this contribution, the proposed model is focused on the Sinusoidal Pulse Width Modulation (SPWM) technique. The switching functions can be written in terms of the complex Fourier series, as follows:

$$
\begin{aligned}
& S_{a}=0.5+2 \sum_{k=1}^{n_{\max }}\left|c_{a\langle k\rangle}\right| \cos \left(k \omega_{0} t+\angle c_{a\langle k\rangle}\right), \\
& S_{b}=0.5+2 \sum_{k=1}^{n_{\max }}\left|c_{b\langle k\rangle}\right| \cos \left(k \omega_{0} t+\angle c_{b\langle k\rangle}\right), \\
& S_{c}=0.5+2 \sum_{k=1}^{n_{\max }}\left|c_{c\langle k\rangle}\right| \cos \left(k \omega_{0} t+\angle c_{c\langle k\rangle}\right),
\end{aligned}
$$

where $n_{\max }$ is the highest harmonic order.

The complex Fourier series coefficients of the switching functions $\left(c_{a\langle k\rangle}, c_{b\langle k\rangle}\right.$, and $\left.c_{c\langle k\rangle}\right)$ strictly depend on the switching times; therefore, the switching functions can be described analytically if the switching times are also described by analytical and explicit expressions in terms of the modulation parameters of the VSC. According to this idea, the next step toward the proposed model is to develop analytical and explicit equations for the computation of the switching times.

\section{Symbolic and Explicit Equations for the Computation of the Switching Times}

The desired voltages at the terminals of the VSC are

$$
\begin{aligned}
& v_{t a}^{\mathrm{ref}}=|V| \cos \left(\omega_{0} t-\theta\right), \\
& v_{t b}^{\mathrm{ref}}=|V| \cos \left(\omega_{0} t-\varphi\right), \\
& v_{t c}^{\mathrm{ref}}=|V| \cos \left(\omega_{0} t-\phi\right) .
\end{aligned}
$$

In particular, for the balanced case, $\varphi=\theta+2 \pi / 3, \phi=$ $\theta-2 \pi / 3$. The switching times can be computed as follows: 


$$
\begin{aligned}
& t_{a\langle k\rangle}= \begin{cases}a_{\langle k\rangle}+\frac{\cos \left(a_{\langle k\rangle} \omega_{0}-\theta\right)}{\sin \left(a_{\langle k\rangle} \omega_{0}-\theta\right) \omega_{0}+(-1)^{k}\left(m_{p} / m_{a}\right)} ; & \theta \in\left(\frac{\pi}{2}, \frac{3 \pi}{2}\right), \\
a_{\langle k+1\rangle}+\frac{\cos \left(a_{\langle k+1\rangle} \omega_{0}-\theta\right)}{\sin \left(a_{\langle k+1\rangle} \omega_{0}-\theta\right) \omega_{0}+(-1)^{k+1}\left(m_{p} / m_{a}\right)} ; & \theta \notin\left(\frac{\pi}{2}, \frac{3 \pi}{2}\right),\end{cases} \\
& t_{b\langle k\rangle}= \begin{cases}a_{\langle k\rangle}+\frac{\cos \left(a_{\langle k\rangle} \omega_{0}-\theta-2 \pi / 3\right)}{\sin \left(a_{\langle k\rangle} \omega_{0}-\theta-2 \pi / 3\right) \omega_{0}+(-1)^{k}\left(m_{p} / m_{a}\right)} ; & \theta \notin\left[\frac{5 \pi}{6}, \frac{11 \pi}{6}\right], \\
a_{\langle k+1\rangle}+\frac{\cos \left(a_{\langle k+1\rangle} \omega_{0}-\theta-2 \pi / 3\right)}{\sin \left(a_{\langle k+1\rangle} \omega_{0}-\theta-2 \pi / 3\right) \omega_{0}+(-1)^{k+1}\left(m_{p} / m_{a}\right)} ; & \theta \in\left[\frac{5 \pi}{6}, \frac{11 \pi}{6}\right],\end{cases} \\
& t_{c\langle k\rangle}= \begin{cases}a_{\langle k\rangle}+\frac{\cos \left(a_{\langle k\rangle} \omega_{0}-\theta+2 \pi / 3\right)}{\sin \left(a_{\langle k\rangle} \omega_{0}-\theta+2 \pi / 3\right) \omega_{0}+(-1)^{k}\left(m_{p} / m_{a}\right)} ; & \theta \notin\left[\frac{\pi}{6}, \frac{7 \pi}{6}\right], \\
a_{\langle k+1\rangle}+\frac{\cos \left(a_{\langle k+1\rangle} \omega_{0}-\theta+2 \pi / 3\right)}{\sin \left(a_{\langle k+1\rangle} \omega_{0}-\theta+2 \pi / 3\right) \omega_{0}+(-1)^{k+1}\left(m_{p} / m_{a}\right)} ; & \theta \in\left[\frac{\pi}{6}, \frac{7 \pi}{6}\right] .\end{cases}
\end{aligned}
$$

Equations (4a)-(4c) can be transformed into a Newton process in order to increase the precision of the switching times:

$$
\begin{aligned}
& t_{a\langle k\rangle}^{\langle i+1\rangle}= \begin{cases}t_{a\langle k\rangle}^{\langle i\rangle}+\frac{\cos \left(t_{a\langle k\rangle}^{\langle i\rangle} \omega_{0}-\theta\right)+(-1)^{k}\left(m_{p} / m_{a}\right)\left(a_{\langle k\rangle}-t_{a\langle k\rangle}^{\langle i\rangle}\right)}{\sin \left(t_{a\langle k\rangle}^{\langle i\rangle} \omega_{0}-\theta\right) \omega_{0}+(-1)^{k}\left(m_{p} / m_{a}\right)} ; & \theta \in\left(\frac{\pi}{2}, \frac{3 \pi}{2}\right), \\
t_{a\langle k\rangle}^{\langle i\rangle}+\frac{\cos \left(t_{a\langle k\rangle}^{\langle i\rangle} \omega_{0}-\theta\right)+(-1)^{k+1}\left(m_{p} / m_{a}\right)\left(a_{\langle k+1\rangle}-t_{a\langle k\rangle}^{\langle i\rangle}\right)}{\sin \left(t_{a\langle k\rangle}^{\langle i\rangle} \omega_{0}-\theta\right) \omega_{0}+(-1)^{k+1}\left(m_{p} / m_{a}\right)} ; & \theta \notin\left(\frac{\pi}{2}, \frac{3 \pi}{2}\right),\end{cases} \\
& t_{b\langle k\rangle}^{\langle i+1\rangle}= \begin{cases}t_{b\langle k\rangle}^{\langle i\rangle}+\frac{\cos \left(t_{b\langle k\rangle}^{\langle i\rangle} \omega_{0}-\theta-2 \pi / 3\right)+(-1)^{k}\left(m_{p} / m_{a}\right)\left(a_{\langle k\rangle}-t_{b\langle k\rangle}^{\langle i\rangle}\right)}{\sin \left(t_{b\langle k\rangle}^{\langle i\rangle} \omega_{0}-\theta-2 \pi / 3\right) \omega_{0}+(-1)^{k}\left(m_{p} / m_{a}\right)} ; & \theta \notin\left[\frac{5 \pi}{6}, \frac{11 \pi}{6}\right], \\
t_{b\langle k\rangle}^{\langle i\rangle}+\frac{\cos \left(t_{b\langle k\rangle}^{\langle i\rangle} \omega-\omega_{0}-2 \pi / 3\right)+(-1)^{k+1}\left(m_{p} / m_{a}\right)\left(a_{\langle k+1\rangle}-t_{b\langle k\rangle}^{\langle i\rangle}\right)}{\sin \left(t_{b\langle k\rangle}^{\langle i\rangle} \omega_{0}-\theta-2 \pi / 3\right) \omega_{0}+(-1)^{k+1}\left(m_{p} / m_{a}\right)} ; & \theta \in\left[\frac{5 \pi}{6}, \frac{11 \pi}{6}\right],\end{cases} \\
& t_{c\langle k\rangle}^{\langle i+1\rangle}= \begin{cases}t_{c\langle k\rangle}^{\langle i\rangle}+\frac{\cos \left(t_{c\langle k\rangle}^{\langle i\rangle} \omega_{0}-\theta+2 \pi / 3\right)+(-1)^{k}\left(m_{p} / m_{a}\right)\left(a_{\langle k\rangle}-t_{c\langle k\rangle}^{\langle i\rangle}\right)}{\sin \left(t_{c\langle k\rangle}^{\langle i\rangle} \omega_{0}-\theta+2 \pi / 3\right) \omega_{0}+(-1)^{k}\left(m_{p} / m_{a}\right)} ; & \theta \notin\left[\frac{\pi}{6}, \frac{7 \pi}{6}\right], \\
t_{c\langle k\rangle}^{\langle i\rangle}+\frac{\cos \left(t_{c\langle k\rangle}^{\langle i\rangle} \omega_{0}-\theta+2 \pi / 3\right)+(-1)^{k+1}\left(m_{p} / m_{a}\right)\left(a_{\langle k+1\rangle}-t_{c\langle k\rangle}^{\langle i\rangle}\right)}{\sin \left(t_{c\langle k\rangle}^{\langle i\rangle} \omega_{0}-\theta+2 \pi / 3\right) \omega_{0}+(-1)^{k+1}\left(m_{p} / m_{a}\right)} ; & \theta \in\left[\frac{\pi}{6}, \frac{7 \pi}{6}\right] .\end{cases}
\end{aligned}
$$

The initial guess for the iterative equations $(5 a)-(5 c)$ is

$$
\begin{aligned}
& t_{a\langle k\rangle}^{\langle 0\rangle}= \begin{cases}a_{\langle k\rangle} ; & \theta \in\left(\frac{\pi}{2}, \frac{3 \pi}{2}\right), \\
a_{\langle k+1\rangle} ; & \theta \notin\left(\frac{\pi}{2}, \frac{3 \pi}{2}\right),\end{cases} \\
& t_{b\langle k\rangle}^{\langle 0\rangle}= \begin{cases}a_{\langle k\rangle} ; & \theta \notin\left[\frac{5 \pi}{6}, \frac{11 \pi}{6}\right], \\
a_{\langle k+1\rangle} ; & \theta \in\left[\frac{5 \pi}{6}, \frac{11 \pi}{6}\right],\end{cases}
\end{aligned}
$$

$$
t_{c\langle k\rangle}^{\langle 0\rangle}= \begin{cases}a_{\langle k\rangle} ; & \theta \notin\left[\frac{\pi}{6}, \frac{7 \pi}{6}\right], \\ a_{\langle k+1\rangle} ; & \theta \in\left[\frac{\pi}{6}, \frac{7 \pi}{6}\right] .\end{cases}
$$

Once the switching instants have been calculated, the switching functions are automatically obtained as follows:

$$
\begin{aligned}
& S_{a\langle k\rangle} \\
& = \begin{cases}\frac{1+(-1)^{k}}{2} ; & \theta \in\left(\frac{\pi}{2}, \frac{3 \pi}{2}\right) ; \forall k=1,2, \ldots, 2 m_{f}+1, \\
\frac{1+(-1)^{k+1}}{2} ; & \theta \notin\left(\frac{\pi}{2}, \frac{3 \pi}{2}\right) ; \forall k=1,2, \ldots, 2 m_{f}+1,\end{cases}
\end{aligned}
$$




$$
\begin{aligned}
& S_{b\langle k\rangle} \\
& = \begin{cases}\frac{1+(-1)^{k}}{2} ; & \theta \notin\left[\frac{5 \pi}{6}, \frac{11 \pi}{6}\right] ; \forall k=1, \ldots, 2 m_{f}+1, \\
\frac{1+(-1)^{k+1}}{2} ; & \theta \in\left[\frac{5 \pi}{6}, \frac{11 \pi}{6}\right] ; \forall k=1, \ldots, 2 m_{f}+1,\end{cases} \\
& S_{c\langle k\rangle} \\
& = \begin{cases}\frac{1+(-1)^{k}}{2} ; & \theta \notin\left[\frac{\pi}{6}, \frac{7 \pi}{6}\right] ; \forall k=1,2,3, \ldots, 2 m_{f}+1, \\
\frac{1+(-1)^{k+1}}{2} ; & \theta \in\left[\frac{\pi}{6}, \frac{7 \pi}{6}\right] ; \forall k=1,2,3, \ldots, 2 m_{f}+1 .\end{cases}
\end{aligned}
$$

The $k$ th state for switching function $S_{x\langle k\rangle}$ takes place from $t_{x\langle k-1\rangle}$ to $t<t_{x\langle k\rangle}$ and so on and so forth, where $x=a, b, c$. By definition, $t_{x\langle 0\rangle}=0$ and $t_{x\left\langle 2 m_{f}+1\right\rangle}=T$ for $x=a, b, c$. For some phase angles as shown in (7a)-(7c), the switching functions contain $2 m_{f}+1$ states because for those angles the state of $S_{x}$ from $t_{x\left\langle 2 m_{f}\right\rangle}$ to $t<T$ is equal to the state of $S_{x}$ from $t_{x\langle 0\rangle}$ to $t<t_{x<1\rangle}$. If one of the phase angles $(\theta, \varphi$, and $\phi)$ is equal to $\pi / 2$ or $3 \pi / 2$, then the associated switching function only has $2 m_{f}$ states in a full fundamental period. Only for this particular case, the last state is different to the first state.

Equations (4a)-(6c) are the general expression for the approximated computation of the switching instants. In (4a)(4c), $t_{a\langle k\rangle}, t_{b\langle k\rangle}$, and $t_{c\langle k\rangle}$ are the $k$ th switching instants for the switching functions of phases $a, b$, and $c$, respectively. In (5a)(5c), $t_{a\langle k\rangle}^{\langle i\rangle}, t_{b\langle k\rangle}^{\langle i\rangle}$, and $t_{c\langle k\rangle}^{\langle i\rangle}$ are the $k$ th switching instants of $S_{a}$, $S_{b}$, and $S_{c}$, respectively, in the $i$ th Newton iteration. $\theta$ is the phase angle of fundamental components of $v_{t a}^{\text {ref }}$ and is within 0 and $2 \pi ; m_{p}$ is the absolute slope of the triangular signal used in the SPWM modulation technique and is equal to $4 m_{f} / T$; $T$ is the fundamental period of the control signals (3a) $-(3 c)$; $m_{f}$ is the frequency modulation ratio; $m_{a}$ is the amplitude modulation ratio for each phase, and this is equal to $|V| / \nu_{\mathrm{dc}}$; $\omega_{0}=2 \pi / T$ is the desired fundamental frequency; and $a_{\langle k\rangle}$ are the instants where the triangular signal is zero and these are equal to $T(k-1) /\left(2 m_{f}\right)$ for $k=1,2, \ldots, 2 m_{f}+1$.

Example 1. In this example, the switching times for the phase $a$ are computed using (4a)-(6c) with $m_{f}=9, m_{a}=0.6, T=$ $1 / 60 \mathrm{~s}$, and $\theta=\pi / 7 \mathrm{rad}$. Figure 2 shows the SPWM process for this case. The number of switching instants in the linear modulation region is always $2 m_{f}$ for each phase; therefore, there are 18 switching instants for the phase $a$.

In Table 1, the switching instants are listed. In the first column the $k$ th switching instant is listed. In the second column the initial guess obtained with (6a) is listed. In the third column the computed switching instants obtained with (4a) are shown, which correspond to the first iteration of (5a). The fourth and fifth columns show the second and third iteration of (5a), respectively.

3.1. Switching Instants for Odd Switching Functions in the Balanced Case. In the case where the switching functions are odd, for example, $m_{f}=$ odd, they have half-wave symmetry;

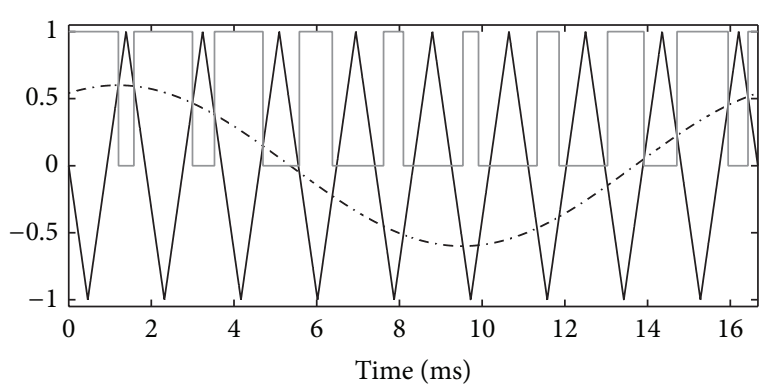

FIGURE 2: SPWM for the phase $a$. Carrier signal (triangular), modulation signal (sinusoidal), and switching function (square).

TABLE 1: Switching times in ms for Example 1.

\begin{tabular}{ccccc}
\hline$k$ & $t_{a\langle k\rangle}^{\langle 0\rangle}=a_{\langle k+1\rangle}$ & $t_{a\langle k\rangle}=t_{a\langle k\rangle}^{\langle 1\rangle}$ & $t_{a\langle k\rangle}^{\langle 2\rangle}$ & $t_{a\langle k\rangle}^{\langle 3\rangle}$ \\
\hline 1 & 0.925925925 & 1.205235647 & 1.2037002983 & 1.203700251 \\
2 & 1.851851851 & 1.575523283 & 1.5770181425 & 1.577018186 \\
3 & 2.777777777 & 2.99403724 & 2.9937987468 & 2.993798739 \\
4 & 3.703703703 & 3.526484448 & 3.5268901728 & 3.526890175 \\
5 & 4.629629629 & 4.697973623 & 4.6979516465 & 4.697951646 \\
6 & 5.555555555 & 5.578734411 & 5.5787334926 & 5.578733492 \\
7 & 6.481481481 & 6.377189967 & 6.3772681085 & 6.377268108 \\
8 & 7.407407407 & 7.617080290 & 7.6164122547 & 7.616412247 \\
9 & 8.333333333 & 8.093941268 & 8.0948938133 & 8.094893827 \\
10 & 9.259259259 & 9.538568981 & 9.5370336316 & 9.537033585 \\
11 & 10.185185185 & 9.908856616 & 9.9103514759 & 9.910351520 \\
12 & 11.111111111 & 11.327837058 & 11.327132080 & 11.327132072 \\
13 & 12.037037037 & 11.859817781 & 11.860223506 & 11.860223508 \\
14 & 12.962962962 & 13.031306957 & 13.031284979 & 13.031284979 \\
15 & 13.888888888 & 13.912067745 & 13.912066825 & 13.912066825 \\
16 & 14.814814814 & 14.710523300 & 14.710601441 & 14.710601441 \\
17 & 15.740740740 & 15.950413624 & 15.949745588 & 15.949745580 \\
18 & 16.666666666 & 16.427274602 & 16.428227146 & 16.428227161 \\
\hline & & & &
\end{tabular}

consequently, all even-numbered harmonics vanish. In addition,

$$
t_{x\left\langle k+m_{f}\right\rangle}=t_{x\langle k\rangle}+\frac{T}{2} ; \quad \forall k=1,2, \ldots, m_{f}, x=a, b, c .
$$

Using half-wave symmetry property, it is only necessary to compute half of the switching times with analytical equations $(4 a)-(6 c)$. For example, from Table 1 , it is easy to show that $t_{a\langle k+9\rangle}=t_{a\langle k\rangle}+1 / 120$ for $k=1,2,3, \ldots, 9$. For the balanced case, the switching times can be computed for one phase, and, for the other phases, these are obtained with 
the appropriate phase angle if $m_{f}$ is odd multiple of three as follows:

$$
\begin{aligned}
& t_{b\langle k\rangle}= \begin{cases}t_{a\left\langle k+4 m_{f} / 3-1\right\rangle}-\frac{2 T}{3} ; & k=1,2, \ldots, \frac{2 m_{f}}{3}+1, \\
t_{a\left\langle k-2 m_{f} / 3-1\right\rangle}+\frac{T}{3} ; & k=\frac{2 m_{f}}{3}+2, \ldots, 2 m_{f},\end{cases} \\
& \forall \theta \notin\left(\frac{\pi}{2}, \frac{11 \pi}{6}\right], \\
& t_{b\langle k\rangle}= \begin{cases}t_{a\left\langle k+4 m_{f} / 3\right\rangle}-\frac{2 T}{3} ; & k=1,2, \ldots, \frac{2 m_{f}}{3}, \\
t_{a\left\langle k-2 m_{f} / 3\right\rangle}+\frac{T}{3} ; & k=\frac{2 m_{f}}{3}+1, \ldots, 2 m_{f},\end{cases} \\
& \forall \theta \in\left[\frac{3 \pi}{2}, \frac{11 \pi}{6}\right] \cup\left(\frac{\pi}{2}, \frac{5 \pi}{6}\right),
\end{aligned}
$$

$$
\begin{aligned}
& t_{b\langle k\rangle} \\
& = \begin{cases}t_{a\left\langle k+4 m_{f} / 3+1\right\rangle}-\frac{2 T}{3} ; & k=1,2, \ldots, \frac{2 m_{f}}{3}-1, \\
t_{a\left\langle k-2 m_{f} / 3+1\right\rangle}+\frac{T}{3} ; & k=\frac{2 m_{f}}{3}, \frac{2 m_{f}}{3}+1, \ldots, 2 m_{f},\end{cases} \\
& \forall \theta \in\left[\frac{5 \pi}{6}, \frac{3 \pi}{2}\right), \\
& t_{c\langle k\rangle}= \begin{cases}t_{a\left\langle k+2 m_{f} / 3-1\right\rangle}-\frac{T}{3} ; & k=1,2, \ldots, \frac{4 m_{f}}{3}+1, \\
t_{a\left\langle k-4 m_{f} / 3-1\right\rangle}+\frac{2 T}{3} ; & k=\frac{4 m_{f}}{3}+2, \ldots, 2 m_{f},\end{cases} \\
& \forall \theta \notin\left[\frac{\pi}{6}, \frac{3 \pi}{2}\right), \\
& t_{c\langle k\rangle}= \begin{cases}t_{a\left\langle k+2 m_{f} / 3\right\rangle}-\frac{T}{3} ; & k=1,2, \ldots, \frac{4 m_{f}}{3}, \\
t_{a\left\langle k-4 m_{f} / 3\right\rangle}+\frac{2 T}{3} ; & k=\frac{4 m_{f}}{3}+1, \ldots, 2 m_{f},\end{cases} \\
& \forall \theta \in\left[\frac{\pi}{6}, \frac{\pi}{2}\right] \cup\left(\frac{7 \pi}{6}, \frac{3 \pi}{2}\right), \\
& t_{c\langle k\rangle}= \begin{cases}t_{a\left\langle k+2 m_{f} / 3+1\right\rangle}-\frac{T}{3} ; & k=1,2, \ldots, \frac{4 m_{f}}{3}-1, \\
t_{a\left\langle k-4 m_{f} / 3+1\right\rangle}+\frac{2 T}{3} ; & k=\frac{4 m_{f}}{3}, \ldots, 2 m_{f},\end{cases} \\
& \forall \theta \in\left(\frac{\pi}{2}, \frac{7 \pi}{6}\right] .
\end{aligned}
$$

If the half-wave symmetry is considered, then (9a)-(9b) are

$$
\begin{array}{r}
t_{b\langle k\rangle} \\
=\left\{\begin{aligned}
t_{a\left\langle k+4 m_{f} / 3-1\right\rangle}-\frac{2 T}{3} ; & k=1,2, \ldots, \\
t_{b\left\langle k-m_{f}\right\rangle}+\frac{T}{2} ; & k=m_{f}+1, m_{f}+2, \ldots, 2 m_{f}, \\
\forall & \forall \notin\left(\frac{\pi}{2}, \frac{11 \pi}{6}\right],
\end{aligned}\right.
\end{array}
$$

$$
\begin{aligned}
& t_{b\langle k\rangle}=\left\{\begin{array}{ll}
t_{a\left\langle k+4 m_{f} / 3\right\rangle}-\frac{2 T}{3} ; & k=1,2,3, \ldots, m_{f}, \\
t_{b\left\langle k-m_{f}\right\rangle}+\frac{T}{2} ; & k=m_{f}+1, \ldots, 2 m_{f},
\end{array} \quad \forall \theta \in\left[\frac{3 \pi}{2}, \frac{11 \pi}{6}\right] \cup\left(\frac{\pi}{2}, \frac{5 \pi}{6}\right),\right. \\
& t_{b\langle k\rangle}= \begin{cases}t_{a\left\langle k+4 m_{f} / 3+1\right\rangle}-\frac{2 T}{3} ; & k=1,2, \ldots, m_{f}, \\
t_{b\left\langle k-m_{f}\right\rangle}+\frac{T}{2} ; & k=m_{f}+1, \ldots, 2 m_{f},\end{cases} \\
& t_{c\langle k\rangle}=\left\{\begin{array}{ll}
t_{a\left\langle k+2 m_{f} / 3-1\right\rangle}-\frac{T}{3} ; & k=1,2, \ldots, m_{f}, \\
t_{c\left\langle k-m_{f}\right\rangle}+\frac{T}{2} ; & k=m_{f}+1, \ldots, 2 m_{f},
\end{array}, \quad \forall \theta \in\left[\frac{5 \pi}{6}, \frac{3 \pi}{2}\right),\right. \\
& t_{c\langle k\rangle}=\left\{\begin{array}{ll}
t_{a\left\langle k+2 m_{f} / 3+1\right\rangle}-\frac{T}{3} ; & k=1,2,3, \ldots, m_{f}, \\
t_{a\left\langle k-m_{f}\right\rangle}+\frac{T}{2} ; & k=m_{f}+1, \ldots, 2 m_{f}, \\
t_{c\left\langle k-m_{f}\right\rangle}+\frac{T}{2} ; & k=m_{f}+1, \ldots, 2 m_{f}, \\
t_{a\left\langle k+2 m_{f} / 3\right\rangle}-\frac{T}{3} ; & k=1,2,3, \ldots, m_{f},
\end{array},\right.
\end{aligned}
$$

With (10a), (10b), only the sixth part $\left(m_{f}\right.$ switching instants) of the total switching instants $\left(6 m_{f}\right)$ has to be computed with (4a)-(4c) or (5a)-(5c) and, consequently, the computational effort is dramatically reduced.

\section{Complex Fourier Series Coefficients of the Switching Functions}

Once switching instants have been analytically calculated, the pulse train of the switching function is automatically obtained with $(7 a)-(7 c)$ in the linear modulation. The complex Fourier series coefficients of the switching function are zero for even harmonic components if $m_{f}=$ odd. On the other hand, the odd complex Fourier series coefficients for $m_{f}=$ odd are

$$
\begin{aligned}
& c_{a\langle k\rangle}=\frac{1}{j \pi k} \sum_{n=1}^{m_{f}}(-1)^{n} e^{-j \omega_{0} k t_{a\langle n\rangle}} \\
& =-\frac{1}{\pi k}\left(\sum_{n=1}^{m_{f}}(-1)^{n} \sin \left(\omega_{0} k t_{a\langle n\rangle}\right)\right. \\
& \left.+j \sum_{n=1}^{m_{f}}(-1)^{n} \cos \left(\omega_{0} k t_{a\langle n\rangle}\right)\right) \\
& \forall \theta \in\left[-\frac{\pi}{2}, \frac{\pi}{2}\right], k=\text { odd }
\end{aligned}
$$




$$
\begin{aligned}
& c_{a\langle k\rangle}=-\frac{1}{j \pi k} \sum_{n=1}^{m_{f}}(-1)^{n} e^{-j \omega_{0} k t_{a\langle n\rangle}} \\
& =\frac{1}{\pi k}\left(\sum_{n=1}^{m_{f}}(-1)^{n} \sin \left(\omega_{0} k t_{a\langle n\rangle}\right)\right. \\
& \left.+j \sum_{n=1}^{m_{f}}(-1)^{n} \cos \left(\omega_{0} k t_{a\langle n\rangle}\right)\right) \\
& \forall \theta \notin\left[-\frac{\pi}{2}, \frac{\pi}{2}\right], k=\text { odd }, \\
& c_{b\langle k\rangle}=\frac{1}{j \pi k} \sum_{n=1}^{m_{f}}(-1)^{n} e^{-j \omega_{0} k t_{b\langle n\rangle}} \\
& =-\frac{1}{\pi k}\left(\sum_{n=1}^{m_{f}}(-1)^{n} \sin \left(\omega_{0} k t_{b\langle n\rangle}\right)\right. \\
& \left.+j \sum_{n=1}^{m_{f}}(-1)^{n} \cos \left(\omega_{0} k t_{b\langle n\rangle}\right)\right) \\
& \forall \theta \in\left[\frac{5 \pi}{6}, \frac{11 \pi}{6}\right], k=\text { odd }, \\
& c_{b\langle k\rangle}=-\frac{1}{j \pi k} \sum_{n=1}^{m_{f}}(-1)^{n} e^{-j \omega_{0} k t_{b\langle n\rangle}} \\
& =\frac{1}{\pi k}\left(\sum_{n=1}^{m_{f}}(-1)^{n} \sin \left(\omega_{0} k t_{b\langle n\rangle}\right)\right. \\
& \left.+j \sum_{n=1}^{m_{f}}(-1)^{n} \cos \left(\omega_{0} k t_{b\langle n\rangle}\right)\right) \\
& \forall \theta \notin\left[\frac{5 \pi}{6}, \frac{11 \pi}{6}\right], k=\text { odd }, \\
& c_{c\langle k\rangle}=\frac{1}{j \pi k} \sum_{n=1}^{m_{f}}(-1)^{n} e^{-j \omega_{0} k t_{c\langle n\rangle}} \\
& =-\frac{1}{\pi k}\left(\sum_{n=1}^{m_{f}}(-1)^{n} \sin \left(\omega_{0} k t_{c\langle n\rangle}\right)\right. \\
& \left.+j \sum_{n=1}^{m_{f}}(-1)^{n} \cos \left(\omega_{0} k t_{c\langle n\rangle}\right)\right) \\
& \forall \theta \in\left[\frac{\pi}{6}, \frac{7 \pi}{6}\right], k=\text { odd }, \\
& c_{c\langle k\rangle}=-\frac{1}{j \pi k} \sum_{n=1}^{m_{f}}(-1)^{n} e^{-j \omega_{0} k t_{c\langle n\rangle}} \\
& =\frac{1}{\pi k}\left(\sum_{n=1}^{m_{f}}(-1)^{n} \sin \left(\omega_{0} k t_{c\langle n\rangle}\right)\right. \\
& \left.+j \sum_{n=1}^{m_{f}}(-1)^{n} \cos \left(\omega_{0} k t_{c\langle n\rangle}\right)\right) \\
& \forall \theta \notin\left[\frac{\pi}{6}, \frac{7 \pi}{6}\right], k=\text { odd } .
\end{aligned}
$$

\section{Case Study}

With the purpose of showing the properties of the proposed formulation of the VSC, a small but complex power system is considered as a case study. This system is periodic, discontinuous, and nonlinear, with a closed-loop control. The test case is shown in Figure 4, where the STATCOM includes the control system based on that described in [18]; 


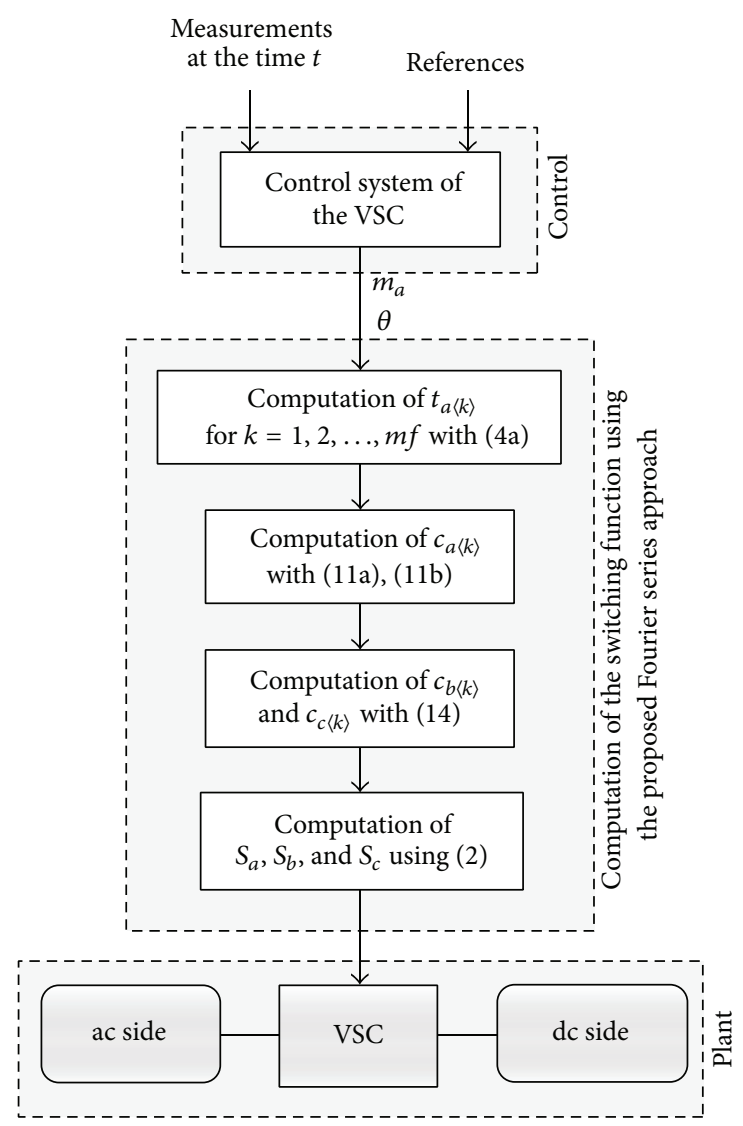

FIGURE 3: Proposed Fourier series model: process for the computation of the switching function using the proposed approach at the time $t$.

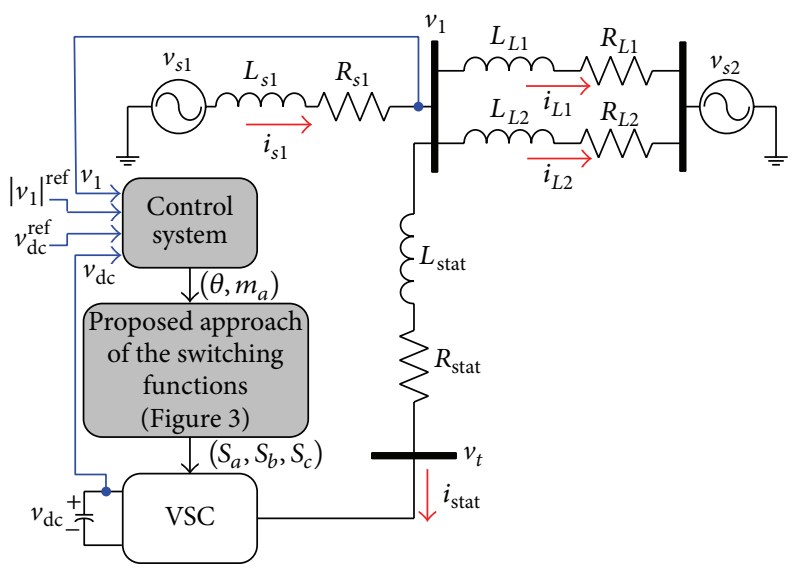

FIGURE 4: Test system including a STATCOM.

Figure 5 shows the control system. The reference line-toline rms voltage is 200 Volts $\left(\left|v_{1}\right|^{\text {ref }}=200 \sqrt{2 / 3}\right)$ at the point of common coupling (PCC); the reference voltage for the dc capacitor is $v_{\mathrm{dc}}^{\text {ref }}=500$ Volts. Table 2 shows the parameters of the test system. It is important to remark that this case study is described by a set of highly nonlinear and discontinuous ordinary differential equations. This case study

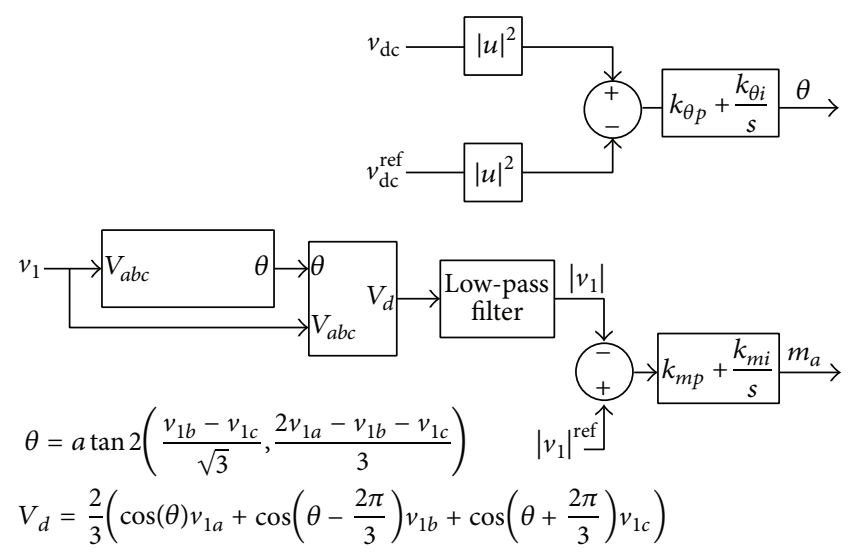

FIGURE 5: STATCOM control system.

TABLE 2: Data of the test system.

\begin{tabular}{l}
$\begin{array}{l}\text { Electric and } \\
\text { control } \\
\text { parameters }\end{array}$ \\
\hline$R_{s 1}=0.1 \Omega$ \\
$L_{s 1}=0.01 \mathrm{H}$ \\
$R_{L 1}=0.2 \Omega$ \\
$L_{L 1}=0.015 \mathrm{H}$ \\
$R_{L 2}=0.15 \Omega$ \\
$L_{L 2}=0.01 \mathrm{H}$ \\
$C_{\mathrm{dc}}=1000 \mu \mathrm{F}$ \\
$R_{\text {stat }}=0.05 \Omega$ \\
$L_{\text {stat }}=0.005 \mathrm{H}$ \\
$r=1 \mathrm{~m} \Omega$ \\
$f=60 \mathrm{~Hz} \quad V_{s 1}=200 \sqrt{\frac{2}{3}}\left[\begin{array}{c}\cos (2 \pi f t) \\
\cos \left(2 \pi f t-\frac{2 \pi}{3}\right) \\
\cos \left(2 \pi f t+\frac{2 \pi}{3}\right)\end{array}\right]$ Volts \\
$K_{m p}=0.004$ \\
$K_{m i}=1.6$ \\
$K_{\theta p}=1.6 \times 10^{-6}$ \\
$K_{\theta i}=6 \times 10^{-5}$
\end{tabular}$\quad\left[\begin{array}{c}\cos (2 \pi f t-0.436) \\
\cos \left(2 \pi f t-\frac{2 \pi}{3}-0.436\right) \\
\cos \left(2 \pi f t+\frac{2 \pi}{3}-0.436\right)\end{array}\right]$ Volts

is first carried out for low switching frequency and then is repeated for a higher switching frequency in order to evaluate the performance of the proposed approach.

5.1. Low Switching Frequency. In this case, the modulation index is $m_{f}=21(1260 \mathrm{~Hz})$. Figure 6 shows a comparison in transient state between Simulink and the proposed model with $n_{\max }=210$ which is equivalent to $12.6 \mathrm{kHz}$ or ten times the switching frequency. Figure 6(a) shows the source current of phase $a\left(i_{s a 1}\right)$, Figure 6(b) shows the STATCOM current of phase $a\left(i_{\text {stat } a}\right)$, and Figure 6(c) shows the voltage across the dc capacitor $\left(v_{\mathrm{dc}}\right)$. Please observe that the obtained solution with Simulink and the proposed model are in very close agreement. However, Simulink requires an integration step of $1 \mu \mathrm{s}$; meanwhile the proposed model requires an integration step of $39.7 \mu \mathrm{s}$. According to the Nyquist-Shannon sampling theorem, the largest integration step must be $1 /(2 \times 12600)$ 


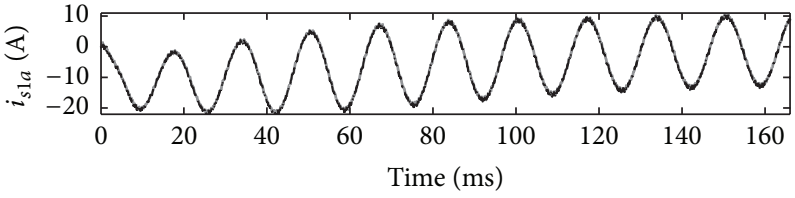

(a)

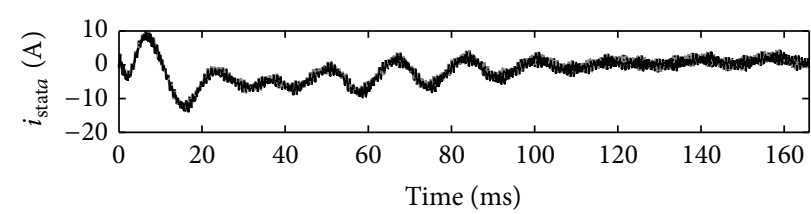

(b)

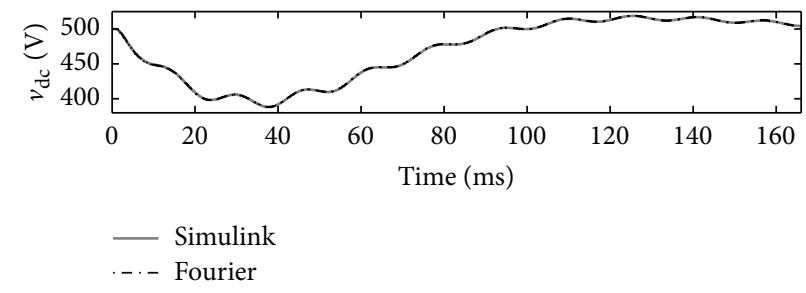

(c)

FIGURE 6: Selected transient waveforms of the test case. (a) The source current of phase $a\left(i_{\text {sal }}\right)$. (b) STATCOM current of phase $a\left(i_{\text {stat } a}\right)$. (c) Voltage across the dc capacitor.

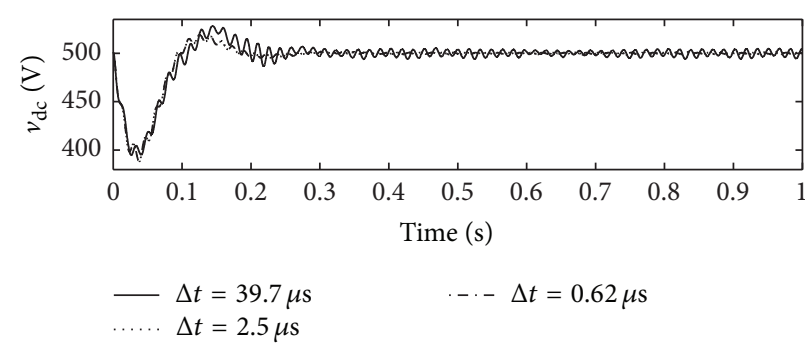

(a)

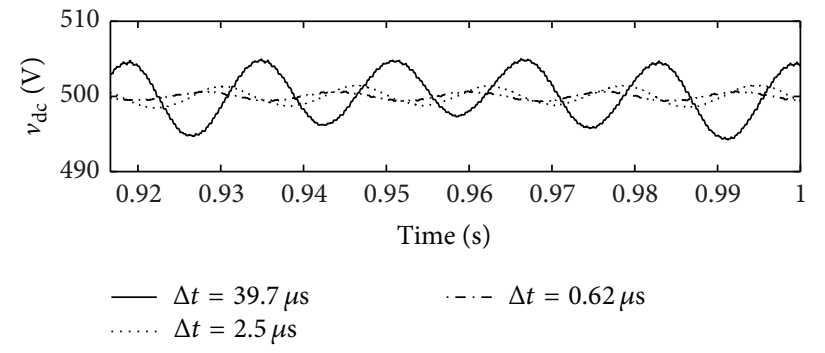

(b)

FIgURE 7: (a) Time evolution of $v_{\mathrm{dc}}$ for two seconds. (b) $v_{\mathrm{dc}}$ for the last five full cycles.

or $39.7 \mu \mathrm{s}$; otherwise, the total harmonic components below $12.6 \mathrm{kHz}$ will not be taken into account in the simulation. In the SPWM, there are around $n_{\max } / 2$ nonzero complex Fourier series coefficients; however, most of them are very small and can be neglected in order to reduce the operations and thus to reduce the computational effort. For this case, there are $n_{\max } / 2$ nonzero complex Fourier series coefficients (odd components); however, there are only 81 dominant harmonic components $\left(>1 \times 10^{-3}\right)$.

The steady state solution comparison is carried out as follows. Firstly, a time domain simulation during one second is carried out in Simulink in order to obtain the steady state solution. Figure 7 (a) shows the obtained solution with Simulink for the voltage at the dc capacitor with three different integration steps. On the other hand, Figure 7(b) shows the last five full cycles of this solution; notice that the solution is still varying regardless of the simulation time [7] and this is highly affected by the integration step. This behavior is due to the numerical error introduced by the discontinuities of the ideal model of the semiconductors [7, 17]. One of the advantages of the proposed formulation is that it can be used in a Newton method, in order to compute the periodic steady state solution and its respective stability. Figure 8 shows the periodic steady state waveforms of $v_{\mathrm{dc}}$ and $i_{\text {stata }}$ for two different integration steps in order to show that the proposed
TABLE 3: Convergence errors for $n_{\max }=210$ and $m_{f}=21$.

\begin{tabular}{llll}
\hline Iteration & \multicolumn{2}{c}{ Error } \\
& $\Delta t=39.7 \mu \mathrm{s}$ & $\Delta t=9.92 \mu \mathrm{s}$ \\
\hline 1 & $5.8314 \times 10^{-4}$ & $5.9212 \times 10^{-4}$ \\
2 & $5.5949 \times 10^{-6}$ & $5.6916 \times 10^{-6}$ \\
3 & $3.4122 \times 10^{-11}$ & $3.2503 \times 10^{-11}$ \\
\hline
\end{tabular}

formulation can be used in simulations with large integration steps. These periodic solutions were computed with a Newton method based on the enhanced numerical differentiation process [1]. Table 3 shows the convergence error of the Newton method for two different integrations steps.

5.2. High Switching Frequency. In practical applications of low power systems, the switching frequency can be up to $100 \mathrm{kHz}$, but, in this case study, we consider a modulation index of $m_{f}=165(9900 \mathrm{~Hz})$; however, the proposed formulation can handle a higher modulation index. For this particular case $\left(m_{f}=165\right)$, there are only 89 dominant harmonic components $\left(>1 \times 10^{-3}\right)$, which hardly represent the $11 \%$ of $n_{\max } / 2$. This consideration reduces almost $90 \%$ of the computation effort. Figure 9 (a) shows the source current 

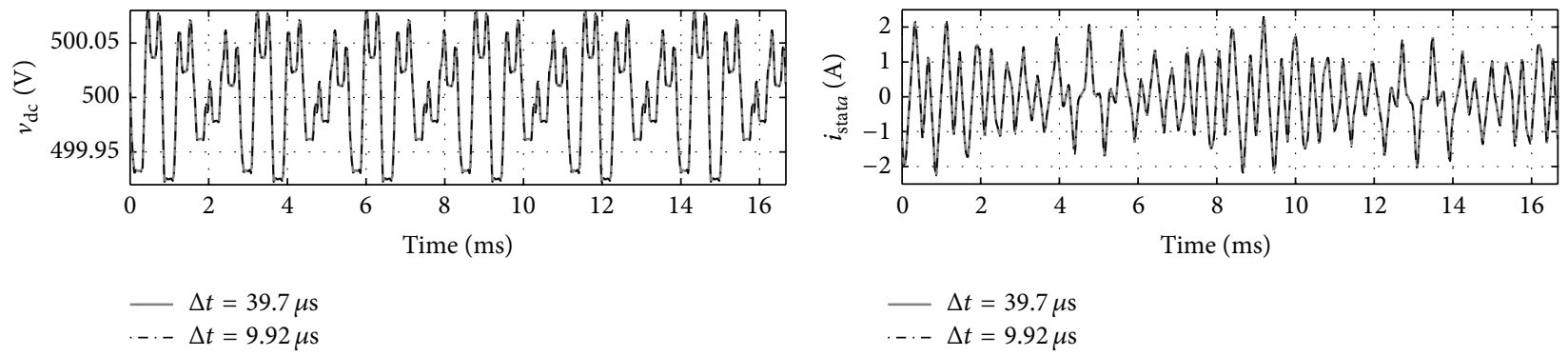

(a)

(b)

FIGURE 8: Steady state solution computed with the proposed model for two different integration steps. (a) Voltage across the dc capacitor $\left(v_{\mathrm{dc}}\right)$. (b) Source current of phase $a\left(i_{\text {sal }}\right)$.

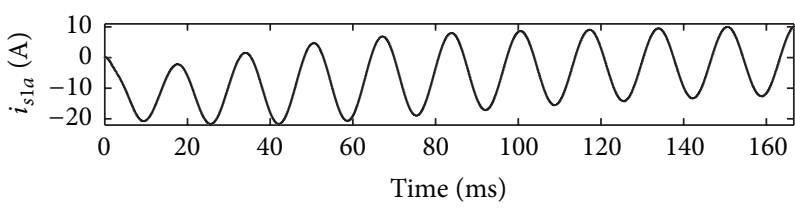

(a)

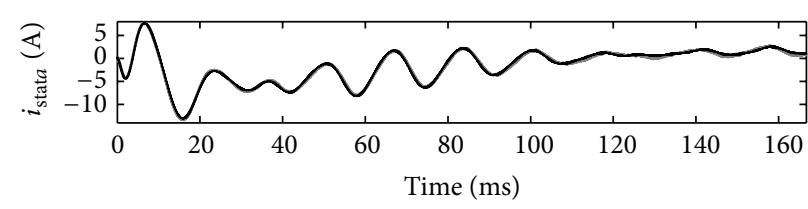

(b)

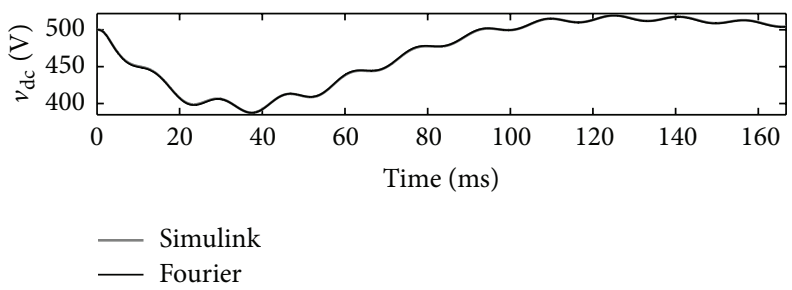

(c)

FIGURE 9: Comparison between Simulink and the proposed model. (a) The source current of phase $a\left(i_{\text {sal }}\right)$. (b) STATCOM current of phase $a\left(i_{\text {stat }}\right)$. (c) Voltage across the dc capacitor.

of phase $a\left(i_{s a 1}\right)$, Figure 9(b) shows the STATCOM current of phase $a\left(i_{\text {stat } a}\right)$, and Figure 9(c) shows the voltage across the dc capacitor $\left(v_{\mathrm{dc}}\right)$. Please observe that the obtained solution with Simulink and the proposed model are practically overlapped. Simulink requires an integration step of $1 \mu$ s and the proposed model requires an integration step of $5.05 \mu \mathrm{s}$; according to the Nyquist-Shannon sampling theorem, this is the largest integration step that can be selected.

Figure 10 shows the periodic steady state waveforms of $v_{\mathrm{dc}}$ and $i_{\text {stata }}$ for two different integration steps in order to show that the proposed formulation can be used in simulations with the largest integration step. These periodic solutions were computed with a Newton method based on the enhanced numerical differentiation process [1]. Table 4 shows the convergence error of the Newton method for two different integrations steps. This case omits the transient comparison since the conclusions are similar to the first case.

It is important to remark that the Newton method has quadratic convergence since the full system is considered in the solution; therefore, this model is able to trace the stability boundary of power networks including VSC-based components. For example, for this operating condition, the maximum Floquet multiplier is 0.9522 , which means stability
TABLE 4: Convergence errors for $n_{\max }=1650$ and $m_{f}=165$.

\begin{tabular}{lll}
\hline Iteration & \multicolumn{2}{c}{ Error } \\
& $\Delta t=5.05 \mu \mathrm{s}$ & $\Delta t=4.17 \mu \mathrm{s}$ \\
\hline 1 & $6.2180 \times 10^{-4}$ & $6.3136 \times 10^{-4}$ \\
2 & $7.6696 \times 10^{-6}$ & $7.8222 \times 10^{-6}$ \\
3 & $3.0805 \times 10^{-11}$ & $3.0823 \times 10^{-11}$ \\
\hline
\end{tabular}

for the system. On the other hand, if the integral gain of the amplitude modulation ratio, $K_{m i}$, is changed from 1.6 to 0.32 , the maximum Floquet multiplier is 1.0023 and now the system is unstable.

Table 5 shows the convergence error of the enhanced numerical differentiation process for this unstable condition. To verify the instability, a time domain simulation is carried out with $K_{m i}=0.32$. Figure 11 shows the time evolution of the voltage across the dc capacitor (a) and the phase portrait of $i_{s 1 a}$ versus $i_{s 1 b}$ (b). From this figure, it is easy to see that the solution is unstable since the phase portrait corresponds to a torus instead of a limit cycle. 


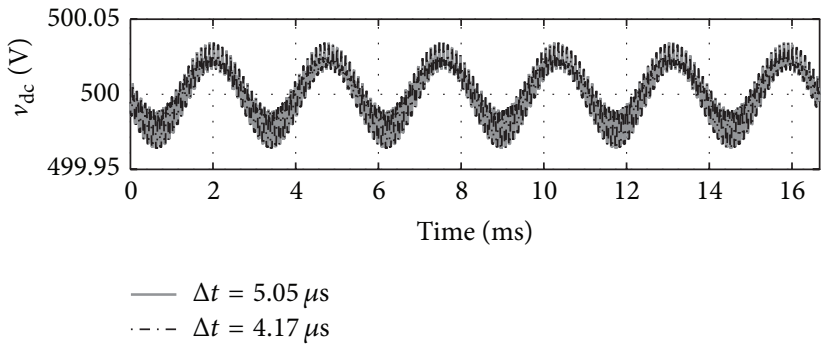

(a)

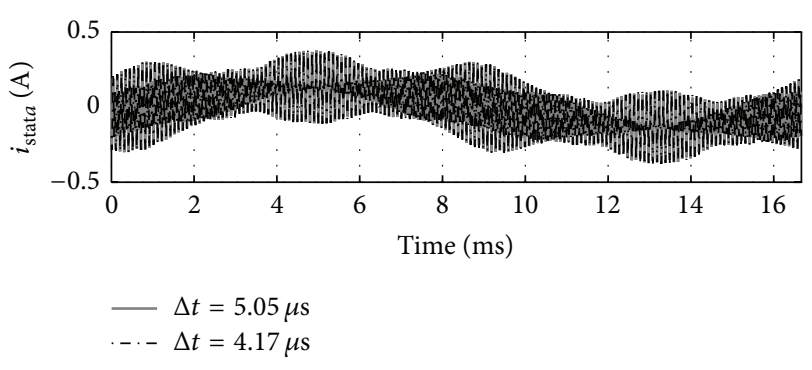

(b)

FIGURE 10: Steady state solution computed with the proposed model for three different integration steps. (a) Voltage across the dc capacitor $\left(v_{\mathrm{dc}}\right)$. (b) Source current of phase $a\left(i_{\text {sal }}\right)$.

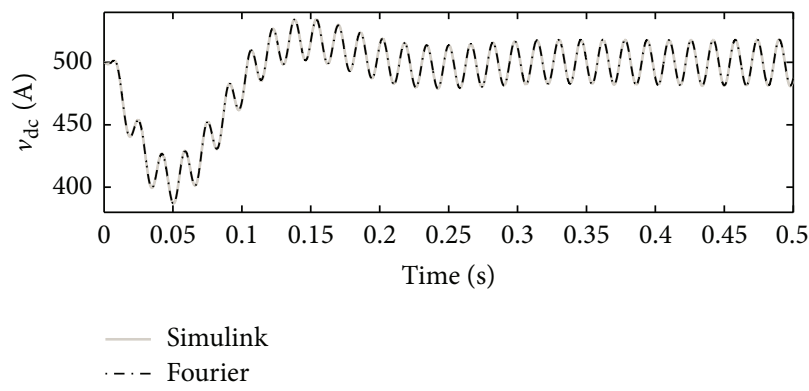

(a)

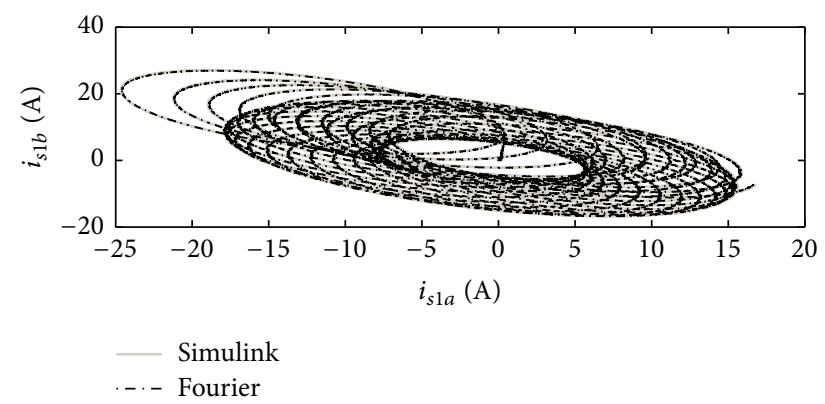

(b)

FIgURE 11: Unstable solution. (a) Time evolution of $v_{\mathrm{dc}}$. (b) Portrait of $i_{s 1 a}$ versus $i_{s 1 b}$.

TABLE 5: Convergence errors for $n_{\max }=1650$ and $m_{f}=165$ (unstable condition).

\begin{tabular}{lcc}
\hline Iteration & \multicolumn{2}{c}{ Error } \\
\hline 1 & $\Delta t=5.05 \mu \mathrm{s}$ & $\Delta t=4.17 \mu \mathrm{s}$ \\
2 & $1.7039 \times 10^{-3}$ & $1.7041 \times 10^{-3}$ \\
3 & $2.3285 \times 10^{-4}$ & $2.3552 \times 10^{-4}$ \\
4 & $1.8926 \times 10^{-8}$ & $1.9204 \times 10^{-8}$ \\
\hline
\end{tabular}

\section{Conclusions}

In this research, a comprehensive modeling of the voltage source converter has been presented. The VSC model was developed in terms of the Fourier series. Additionally, analytical and explicit equations for the computation of the switching times have been obtained and, based on these equations, a set of analytical equations for the computation of the complex Fourier series coefficients of the switching functions was developed. The balanced condition and the properties of the Fourier series for $m_{f}$ equal to odd multiple of three have been exploited in order to compute the three switching functions only with the sixth part of the total switching instants.

Also, the developed model based on the Fourier series representation of the switching functions is suitable for the application of fast time domain methods. In the case of this contribution, a Newton method was applied in order to obtain the periodic steady state solution of a power system in an efficient way. The power system used in the case studies includes nonlinear components with SPWM VSC and with closed-loop control.

In order to prove the benefits of the proposed model, a case study including a STATCOM with a control system has been analyzed. A very important characteristic of the proposed approach has been observed; that is, it is possible to consider only the dominant harmonic components in the solution, which means higher efficiency. For instance, in the second case study, the total harmonic components were 1650; however, only 89 dominant complex Fourier series coefficients were employed in both the transient and the steady state solutions.

In summary, the VSC model developed in this contribution offers the following advantages.

(i) This proposal reaches the periodic steady state solution of a power system in an efficient way by using a Newton method.

(ii) A system that is typically discontinuous is transformed into a continuous one with all its advantages.

(iii) After the transformation, the nonlinear discontinuous system reaches a nonlinear and continuous form including the harmonic information of interest. 


\section{Conflict of Interests}

The authors declare that there is no conflict of interests regarding the publication of this paper.

\section{Acknowledgments}

The authors want to acknowledge the Universidad Autónoma de San Luis Potosí (UASLP) through the Facultad de Ingeniería and the Facultad de Ingeniería Eléctrica of the Universidad Michoacana de San Nicolás de Hidalgo (UMSNH) for the facilities granted to carry out this research. The authors want to thank the project FORDECYT 190966 for the facilities granted to carry out this research.

\section{References}

[1] J. Segundo-Ramírez and A. Medina, "An enhanced process for the fast periodic steady state solution of nonlinear systems by poincaré map and extrapolation to the limit cycle," International Journal of Nonlinear Sciences and Numerical Simulation, vol. 11, no. 8, pp. 661-670, 2010.

[2] M. P. Bahrman, J. G. Johansson, and B. A. Nilsson, "Voltage source converter transmission technologies: the right fit for the application," in Proceedings of the IEEE Power Engineering Society General Meeting, vol. 3, pp. 1840-1847, IEEE, July 2003.

[3] A. Yazdani and R. Iravani, Voltage-Sourced Converters in Power Systems, Wiley, New York, NY, USA, 2010.

[4] Y. Wei, Q. He, Y. Sun, and C. Ji, "Improved power flow algorithm for VSC-HVDC system based on high-order Newton-type method," Mathematical Problems in Engineering, vol. 2013, Article ID 235316, 10 pages, 2013.

[5] X. Jiang, X. Fang, J. H. Chow et al., "A novel approach for modeling voltage-sourced converter-based FACTS controllers," IEEE Transactions on Power Delivery, vol. 23, no. 4, pp. 25912598, 2008.

[6] M. P. Bahrman, J. G. Johansson, and B. A. Nilsson, "Voltage source converter transmission technologies: the right fit for the application," in Proceedings of the IEEE Power Engineering Society General Meeting, pp. 1840-1847, Ontario, Canada, July 2003.

[7] J. Segundo-Ramírez and A. Medina, "Modeling of FACTS devices based on SPWM VSCs," IEEE Transactions on Power Delivery, vol. 24, no. 4, pp. 1815-1823, 2009.

[8] J. Segundo-Ramírez, E. Bárcenas-Bárcenas, A. Medina, and V. Cárdenas, "Steady-state and dynamic state-space model for fast and efficient solution and stability assessment of ASDs," IEEE Transactions on Industrial Electronics, vol. 58, no. 7, pp. 28362847, 2011.

[9] J. Segundo and A. Medina, "Periodic steady-state solution of electric systems including UPFCs by extrapolation to the limit cycle," IEEE Transactions on Power Delivery, vol. 23, no. 3, pp. 1506-1512, 2008.

[10] J. Segundo-Ramírez, A. Medina, A. Ghosh, and G. Ledwich, "Stability boundary analysis of the dynamic voltage restorer in weak systems with dynamic loads," International Journal of Circuit Theory and Applications, vol. 40, no. 6, pp. 551-569, 2012.

[11] J. Segundo-Ramirez and A. Medina, "Periodic steady state solution of FACTS devices based on SPWM VSCs," in Proceedings of the IEEE International Symposium on Industrial Electronics (ISIE '09), pp. 1644-1649, Seoul, Republic of Korea, July 2009.
[12] R. Peña, A. Medina, and O. Anaya-Lara, "Steady-state solution of fixed-speed wind turbines following fault conditions through extrapolation to the limit cycle," IETE Journal of Research, vol. 57, no. 1, pp. 12-19, 2011.

[13] P. W. Lehn, "Exact modeling of the voltage source converter," IEEE Transactions on Power Delivery, vol. 17, no. 1, pp. 217-222, 2002.

[14] P. W. Lehn, "Direct harmonic analysis of the voltage source converter," IEEE Transactions on Power Delivery, vol. 18, no. 3, pp. 1034-1042, 2003.

[15] K. L. Lian and P. W. Lehn, "Steady-state solution of a voltagesource converter with full closed-loop control," IEEE Transactions on Power Delivery, vol. 21, no. 4, pp. 2071-2081, 2006.

[16] K. L. Lian and M. Syai'in, "Steady-state solutions of a voltage source converter with $d q$-frame controllers by means of the time-domain method," IEEJ Transactions on Electrical and Electronic Engineering, vol. 9, no. 2, pp. 165-175, 2014.

[17] J. Vlach, J. M. Wojciechowski, and A. Opal, "Analysis of nonlinear networks with inconsistent initial conditions," IEEE Transactions on Circuits and Systems I: Fundamental Theory and Applications, vol. 42, no. 4, pp. 195-200, 1995.

[18] B. Mahyavanshi and G. Radman, "A study of interaction between dynamic load and STATCOM," in Proceedings of the 38th Southeastern Symposium on System Theory, pp. 392-396, Cookeville, Tenn, USA, March 2006. 


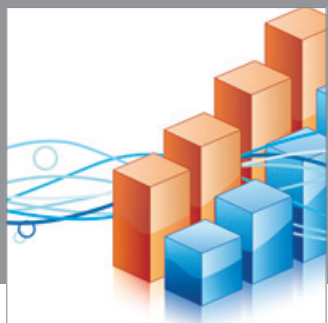

Advances in

Operations Research

mansans

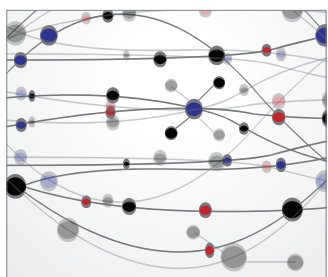

The Scientific World Journal
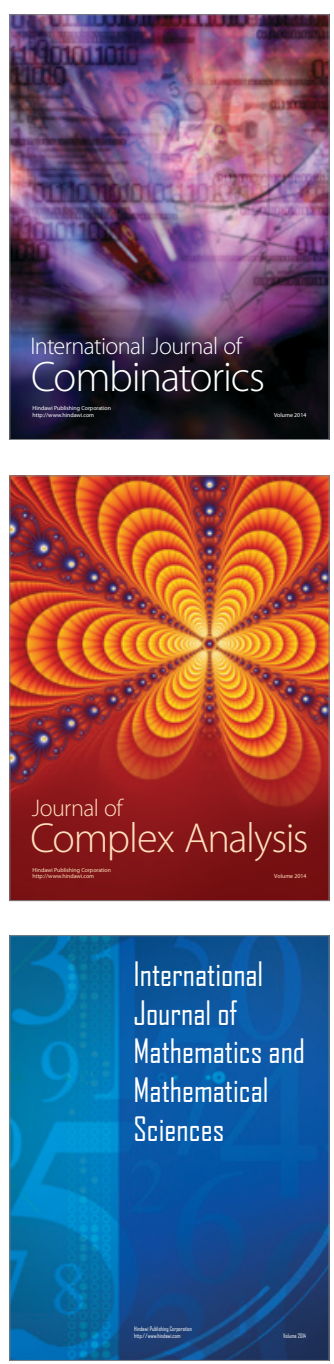
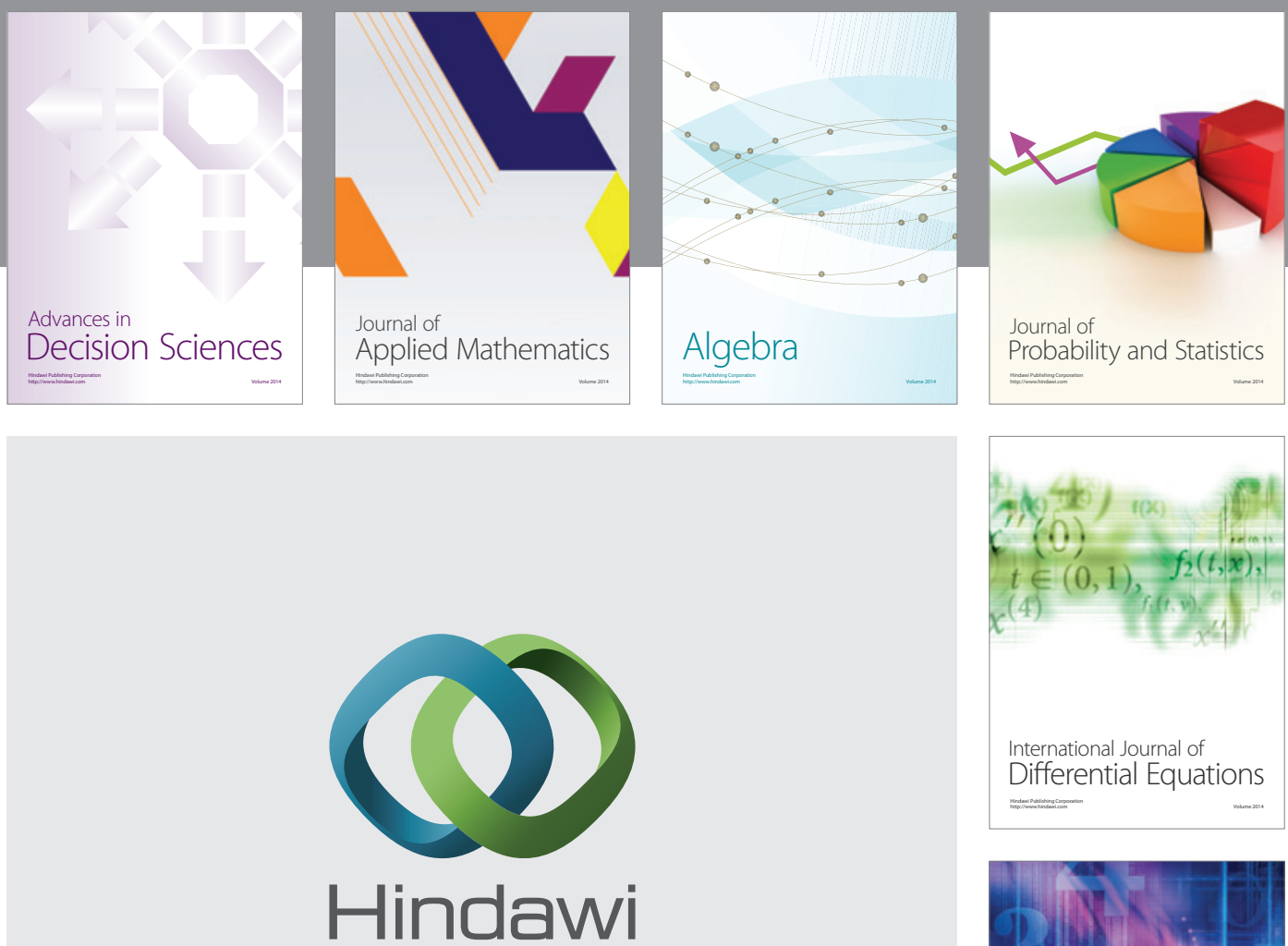

Submit your manuscripts at http://www.hindawi.com
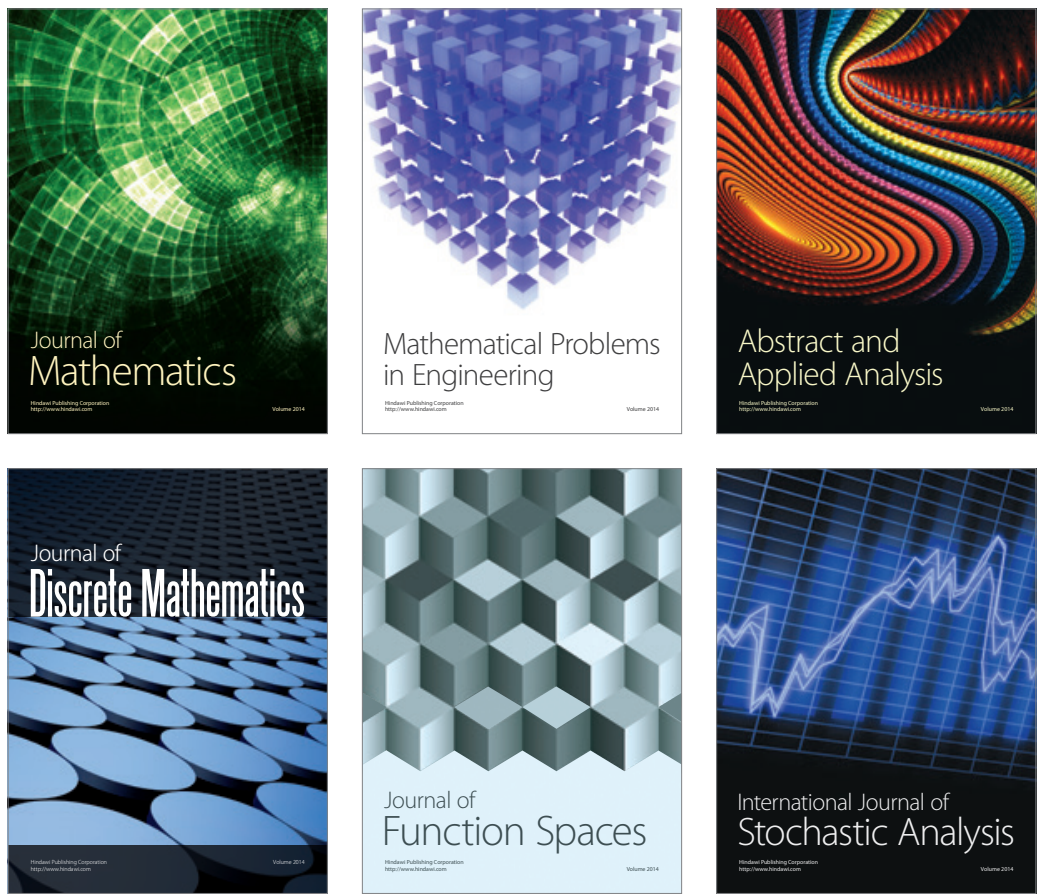

Journal of

Function Spaces

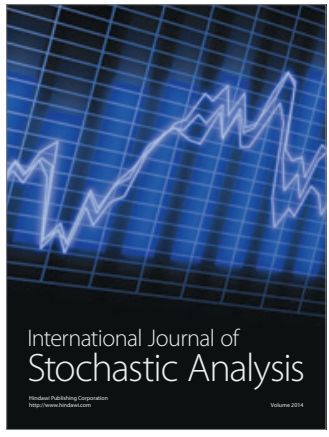

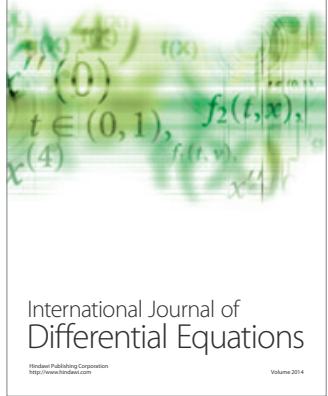
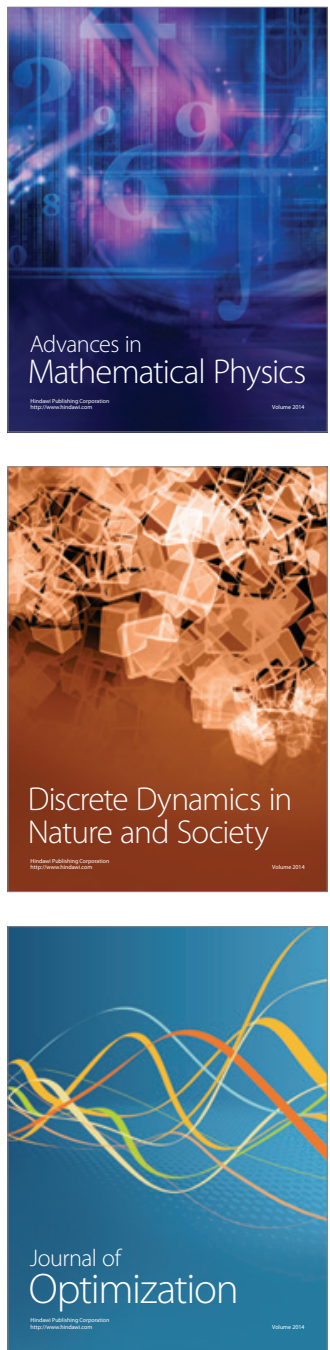